\title{
Unfolded protein response, a link between endometrioid ovarian carcinoma and endometriosis: A pilot study
}

\author{
ANDREA CIAVATTINI $^{1}$, GIOVANNI DELLI CARPINI ${ }^{1}$, MATTEO SERRI $^{1}$, ALESSANDRA TOZZI ${ }^{1}$, \\ FRANCESCA LEONI $^{2}$, EUGENIA DI LORETO ${ }^{1}$ and FRANCA SACCUCCI ${ }^{2}$ \\ ${ }^{1}$ Woman's Health Sciences Department, Gynecologic Section, Polytechnic University of Marche, Ancona I-60123; \\ ${ }^{2}$ Department of Biochemistry, Biology and Genetics, Polytechnic University of Marche, Ancona I-60121, Italy
}

Received December 11, 2017; Accepted June 28, 2018

DOI: $10.3892 / \mathrm{ol} .2018 .9256$

\begin{abstract}
The aim of the present study was to analyze the expression profile of unfolded protein response (UPR) genes in endometrioid ovarian carcinoma and to evaluate its possible involvement in the neoplastic progression of endometriosis. An experimental retrospective pilot study was conducted on women with a diagnosis of endometrioid ovarian carcinoma at FIGO stage IA, ovarian endometriotic cysts or healthy subjects without a previous diagnosis of endometriosis. The expression profiles of UPR genes (ATF6, GRP78, CHOP and XBP1) were compared among ovaries with endometrioid ovarian cancer, endometriotic ovarian cysts, healthy contralateral ovaries and eutopic and healthy endometrial tissues. A significantly higher expression of ATF6 and GRP78 was detected in the affected ovaries in comparison with the healthy contralateral ovaries, while CHOP and XBP1 exhibited a significantly lower expression. XBP1 was overexpressed in endometrial tissues and its expression gradually decreased in endometriosis cysts and endometrioid ovarian carcinoma. These results support the hypothesis that alterations in the UPR genes CHOP and XBP1 are involved in the neoplastic progression of endometrioid ovarian cancer and are acquired following ovarian localization of ectopic endometrial cells.
\end{abstract}

Correspondence to: Professor Andrea Ciavattini, Woman's Health Sciences Department, Gynecologic Section, Polytechnic University of Marche, Via F. Corridoni 11, Ancona I-60123, Italy

E-mail: ciavattini.a@libero.it

Abbreviations: ATF6, activating transcription factor 6; CHOP, CCAAT/-enhancer-binding protein homologous protein; ER, endoplasmic reticulum; GRP78, glucose-regulated protein 78; IRE1, inositol-requiring enzyme 1; PERK, protein kinase R (PKR)-like endoplasmic reticulum kinase; UPR, unfolded protein response; XBP1, spliced X-box binding protein 1

Key words: unfolded protein response, endometriosis, endometrioid ovarian cancer, endometrioma

\section{Introduction}

Endometriosis is an estrogen-dependent chronic inflammatory disorder due to the presence of endometrial tissue outside the uterine cavity (1), and it affects 6-10\% of women of childbearing age; however, this percentage increases to $17 \%$ among infertile women and $40-60 \%$ of women suffering from chronic pelvic pain (2-7). There is solid evidence that endometriosis-affected patients have a doubled risk of ovarian cancer, particularly of the endometrioid and clear-cell histology, and this association is also supported by molecular and histological evidence (5-12).

Although the hypothesis that ovarian cancers develop from 'neometaplasia' of the ovarian surface mesothelium cannot be ruled out (13), there is currently compelling morphological and molecular evidence that any ovarian carcinoma subtype may originate from Müllerian epithelium present in the pelvis, which involves the ovary only at a later stage (12). According to this hypothesis, endometrioid carcinoma and clear-cell carcinoma may originate from ectopic endometrial tissue localized in the ovary (endometriotic cyst or endometrioma). To date, the exact mechanism by which this malignant transformation occurs has not been clearly determined, and further studies are required to elucidate the mechanisms underlying this transformation.

The unfolded protein response (UPR) is a system by which the endoplasmic reticulum (ER) responds to endogenous or exogenous stress (14), and is activated by the accumulation of misfolded proteins in the ER lumen (14). Recent studies have revealed the role of UPR in neoplastic transformation, since it promotes cell survival in a hypoxic environment and plays a protective role against cell death caused by ER stress (15). Cell survival in a hypoxic environment is promoted by attenuation of pro-apoptotic signals, cellular metabolism changes and stimulation of neo-angiogenesis. The activation of UPR may promote cell survival or stimulate apoptosis, depending on the context. This response occurs via the activation of ER transmembrane receptors, namely protein kinase $\mathrm{R}$ (PKR)-like endoplasmic reticulum kinase (PERK), activating transcription factor $6(\mathrm{ATF} 6 \alpha)$ and inositol-requiring enzyme 1-spliced X-box binding protein 1 (IRE1 $\alpha$-XBP1). Under acute stress conditions, the UPR system enhances the ability to fold in order to cope with an increase in protein synthesis, which helps cancerous cells survive (15). Under 
conditions of chronic ER stress, if the normal function of the cell is not restored within a certain time span or the disruption is prolonged (15), normal cells undergo apoptosis-mediated cell death, while cancer cells activate strategies to neutralize the apoptotic stimulus; in normal cells, there is an attenuation of the ATF $6 \alpha$ and IRE1 $\alpha$-XBP1 pathways, with an activation of an apoptotic stimulus mediated by CCAAT/enhancerbinding protein homologous protein (CHOP) (15). Conversely, cancer cells exhibit a constitutive activation of IRE1 $\alpha$-XBP1, or glucose-regulated protein 78 (GRP78)/BIP overexpression, with anti-apoptotic effects. Although the UPR molecular pathway has been studied in several tumor models, its role in the pathogenesis of endometrioid ovarian carcinoma has not yet been fully elucidated.

Therefore, this pilot study was conducted with the aim of analyzing the expression profile of UPR genes in endometrioid ovarian carcinoma and to evaluate its possible involvement in the neoplastic progression of endometriosis.

\section{Materials and methods}

The present study was conducted on women of childbearing age with a histopathological diagnosis of International Federation of Gynecology and Obstetrics (FIGO) stage IA (16) endometrioid ovarian carcinoma, who underwent complete surgical staging (peritoneal washing, bilateral salpingooophorectomy, hysterectomy, multiple peritoneal biopsies of all abdominal fields, infracolic omentectomy, and pelvic and para-aortic lymph node dissection up to the renal veins) (17) between January 2010 and December 2015 at the Woman's Health Sciences Department, Gynecologic Section-Università Politecnica delle Marche (Ancona, Italy), retrospectively recruited from our database.

Patient studies. In order to evaluate the possible involvement of the UPR pathway in the oncogenesis of ovarian endometrioid carcinoma, we compared the expression of ATF6, GRP78, CHOP (also referred to as DNA damage-inducible transcript 3, DDIT3) and XBP1 from a sample of ovary affected by endometrioid ovarian carcinoma and from a sample of the healthy contralateral ovary of the same patient. Subsequently, we compared the UPR gene expression between endometriotic cysts and eutopic healthy endometrial tissues, with the healthy ovary as reference.

Samples of endometriotic ovarian cysts and eutopic endometrium were obtained from patients undergoing laparoscopic surgery for endometrial cyst removal following diagnosis of moderate/severe endometriosis. None of the women were pregnant at the time of surgery. In this subgroup of women, we collected two types of tissue: a portion of the cyst following laparoscopic removal, and a sample of endometrial tissue following hysteroscopic biopsy. All the tissues were stored at $-80^{\circ} \mathrm{C}$.

The samples of healthy endometrial tissue were collected from women of childbearing age, without previous diagnosis of endometriosis, undergoing hysteroscopy for in vitro fertilization due to male infertility. All the women underwent diagnostic laparoscopy that ruled out endometriosis. A sample of endometrial tissue was obtained by hysteroscopic biopsy.
Healthy women and endometriosis patients were consecutively enrolled from January 2016 onwards and, in order to avoid the influence of confounding factors, patients with previous diagnosis of hypertension, diabetes mellitus, renal disease, proteinuria, cardiovascular, hepatic, endocrine (thyroid abnormalities, prolactin imbalance, polycystic ovary syndrome) and metabolic disorders, smokers, and individuals prone to alcohol abuse were excluded from both groups. None of the included subjects had taken any medications or hormone therapy during the 3 months preceding surgery (progestin, oral contraceptives, danazol or gonadotropin-releasing hormone agonists).

The present study was conducted in accordance with The Code of Ethics of the World Medical Association (Declaration of Helsinki). All participants signed an informed consent, granting their permission to tissue sampling and data collection. The study protocol was approved by the local Ethics Committee (Marche Regional Ethics Committee, Riuniti di Ancona Hospital, Italy).

Preparation of paraffin-embedded ovarian tissue samples. Total RNA was isolated from paraffin-embedded ovarian tissues using the PureLink ${ }^{\mathrm{TM}}$ FFPE Total RNA Isolation kit (cat. no. K156002; Life Technologies; Thermo Fisher Scientific, Inc., Waltham, MA, USA) that specifically purifies RNA from paraffin-embedded tissues without the use of chemical solvents for deparaffinization. Total RNA was quantified, and the purity of the preparation was tested using NanoDrop (Thermo Fisher Scientific, Inc.): Briefly, $1 \mu \mathrm{l}$ of purified RNA was placed on the instrument, 260 and 260/230 $\mathrm{nm}$ ratio wavelength was measured and the instrument software then calculated the amount of purified RNA. Reverse transcription to cDNA was performed using Advanced iScript cDNA synthesis kit for RT-qPCR (cat. no. 1725038; Bio-Rad Laboratories, Inc., Hercules, CA, USA). The reverse transcription was performed by preparing a reaction mixture containing $\sim 1 \mu \mathrm{g}$ RNA and random primers provided by the kit. As the RNA obtained from paraffin-embedded samples is typically very fragmented, the efficacy of the amplification reaction by qPCR is generally low; to avoid that, cDNA was pre-amplified using SsoAdvanced ${ }^{\mathrm{TM}}$ PreAmp Supermix (cat. no. 1725160; Bio-Rad Laboratories, Inc.) in conjunction with a pool of primers of target genes.

Preparation of frozen endometrial tissue samples. The purification of total RNA collected from $\sim 30 \mathrm{mg}$ of frozen $\left(-80^{\circ} \mathrm{C}\right)$ endometrial tissues was performed using SV Total RNA Isolation System kit (Z3101; Promega Corporation, Madison, WI, USA), using diethyl pyrocarbonate-treated equipment. Total RNA was quantified, and the purity of the preparation was tested using NanoDrop. Reverse transcription to cDNA was performed with the ImProm-II ${ }^{\mathrm{TM}}$ Reverse Transcription System kit (cat. no. A3800) using $2 \mu \mathrm{g}$ RNA and random primers (cat. no. C1181) (both from Promega Corporation.

Gene expression analysis by $q$ PCR.qPCR was performed using SsoAdvanced $^{\text {TM }}$ SYBR $^{\circledR}$-Green SuperMix (cat. no. 1725271; Bio-Rad Laboratories, Inc.), in a CFX96 thermocycler (Bio$\mathrm{Rad})$. The reaction conditions are summarized as follows: $95^{\circ} \mathrm{C}$ for $30 \mathrm{sec}$, and 40 cycles at $95^{\circ} \mathrm{C}$ for $30 \mathrm{sec}$, and at $60^{\circ} \mathrm{C}$ 
Table I. Primer sequences of the analyzed genes.

\begin{tabular}{lll}
\hline Gene & \multicolumn{1}{c}{ Forward } & \multicolumn{1}{c}{ Reverse } \\
\hline ATF6 & \multicolumn{1}{c}{ TTCCTCCACCTCCTTGTCAG } \\
TGPTAAAGATGAGCGGGTGG & TGCCTACCAAGAAGTCTCAGA & TCCATCCTCGAAGTTCATGA \\
GRP78 & CTGAGTCCGCAGCAGGTG 1 & ACGAGGAGCAGGAGGAATTC \\
GAPDH & TCCACTGGCGTCTTCACC & CCAAGTTGTCCAGAATGCCC \\
& & GGCAGAGATGATGACCCTTTT
\end{tabular}

for $30 \mathrm{sec}$. Melting curves were analyzed after the reaction to assess the specificity of the amplification products; the curves obtained showed no evidence of dimers or non-specific signals. The primers used for the study were designed using Primer 3 software. The primer sequences are listed in Table I.

Gene expression was reported as fold-change of expression in the pathological ovarian tissues compared with control (healthy contralateral ovary), setting 1 as the control reference value. Relative gene expression was calculated using the $\Delta \Delta \mathrm{Cq}$ method (18), converted for statistical purposes to relative expression ratio using the $2^{-\Delta \Delta \mathrm{Cq}}$ formula. All gene expression data were normalized to the expression of the endogenous reference gene, glyceraldehyde-3-phosphate dehydrogenase.

Statistical analysis. Statistical software SPSS v.20.0 (SPSS, Inc., Chicago, IL, USA) was used for data analysis. Foldchange expression of UPR genes was presented as the mean \pm standard error of the mean. The one-sample signedrank sum test was used for comparison with reference tissues (healthy ovary, reference value $=1$ ). The Mann-Whitney test for independent samples was used for comparison of UPR genes fold-change expression between endometriotic cysts and endometrioid carcinoma of the ovary. The Kruskal-Wallis test with the Conover-Iman post hoc test was used for the comparisons among endometriotic cysts, eutopic endometrium and healthy endometrium. A P-value of $<0.05$ was considered to indicate statistically significant differences.

\section{Results}

The UPR gene expression was examined in tissue samples derived from a total of i) 6 patients diagnosed with FIGO stage IA endometrioid ovarian carcinoma who met the inclusion criteria; ii) the first 6 patients consecutively diagnosed with ovarian endometriotic cysts; and iii) 6 healthy patients without previous diagnosis of endometriosis.

Endometrioid carcinoma of the ovary vs. contralateral healty ovary. We first compared the UPR gene expression between endometrioid carcinoma of the ovary and the contralateral healthy ovary of the same patient. A significantly higher expression of ATF6 (fold-change: $4.4 \pm 0.7, \mathrm{P}=0.01$ ) and GRP78 (fold-change: $1.9 \pm 0.2, \mathrm{P}=0.02$ ) was observed in the affected ovary compared with the healthy contralateral ovary, while CHOP and XBP1 exhibited significantly lower expression (fold-change: $0.3 \pm 0.1, \mathrm{P}<0.001$ and $0.5 \pm 0.02, \mathrm{P}<0.001$, respectively) (Fig. 1).

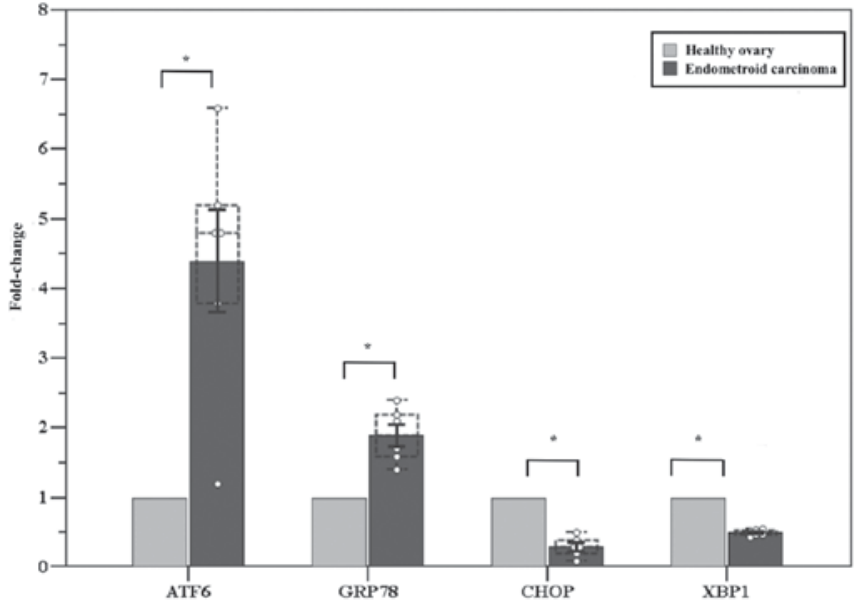

Figure 1. Gene expression analysis of UPR genes in endometrioid carcinoma of the ovary and contralateral healthy ovary. Six patients with FIGO IA endometrioid carcinoma of the ovary (carcinoma and healthy ovary). Solid error bars express the mean \pm standard error of the mean. The dotted error bars indicate the median, the 25 and 75th percentiles and the lowest and highest values. One-sample signed-rank sum test; ${ }^{*} \mathrm{P}<0.05$. UPR, unfolded protein response; FIGO, International Federation of Gynecology and Obstetrics; SEM, standard error of the mean.

Endometriotic cysts vs. healthy ovary vs. endometrioid carcinoma of the ovary. We subsequently evaluated the UPR gene expression in endometriotic cysts compared with healthy ovary and endometrioid carcinoma of the ovary. The expression of ATF6 and GRP78 was significantly higher in the endometriotic cysts compared with that in the healthy ovary (fold-change: $5.2 \pm 0.5, \mathrm{P}<0.001$ and $14.6 \pm 2.2, \mathrm{P}=0.002$, respectively). $\mathrm{CHOP}$ expression in endometriotic cysts was similar to that in the healthy ovary (fold-change: $0.8 \pm 0.1, \mathrm{P}=0.06$ ), while XBP1 was overexpressed (fold-change: $4.2 \pm 0.2, \mathrm{P}<0.001$ ). The comparison between endometriotic cysts and endometrioid carcinoma of the ovary is presented in Table II: ATF6 expression was similar between the two tissues, while GRP78, CHOP and XBP1 were more highly expressed in endometriotic cysts (Table II).

Endometriotic cysts vs. eutopic endometrium vs. healthy endometrium vs. healthy ovary. The fold-change in the expression of ATF6 was significantly higher in endometriotic cysts, eutopic endometrium and healthy endometrium compared with that in healthy ovarian tissue $(5.0 \pm 0.5, \mathrm{P}=0.03 ; 7.5 \pm 1.5$, $\mathrm{P}=0.03$; and $5.8 \pm 1.5, \mathrm{P}=0.03$, respectively), without significant 
Table II. Fold-change gene expression analysis of unfolded protein response genes in endometriosic cysts and endometrioid carcinoma of the ovary.

\begin{tabular}{lccc}
\hline Gene & $\begin{array}{c}\text { Endometriosic } \\
\text { cysts }\end{array}$ & $\begin{array}{c}\text { Endometrioid } \\
\text { carcinoma }\end{array}$ & P-value \\
\hline ATF6 & $5.2 \pm 0.5$ & $4.4 \pm 0.7$ & 0.42 \\
GRP78 & $14.6 \pm 2.2$ & $1.9 \pm 0.4$ & $<0.01$ \\
CHOP & $0.8 \pm 0.1$ & $0.3 \pm 0.1$ & $<0.01$ \\
XBP1s & $4.2 \pm 0.2$ & $0.5 \pm 0.3$ & $<0.01$ \\
\hline
\end{tabular}

Data are presented as the mean \pm standard error of the mean. A MannWhitney test was used for independent samples.

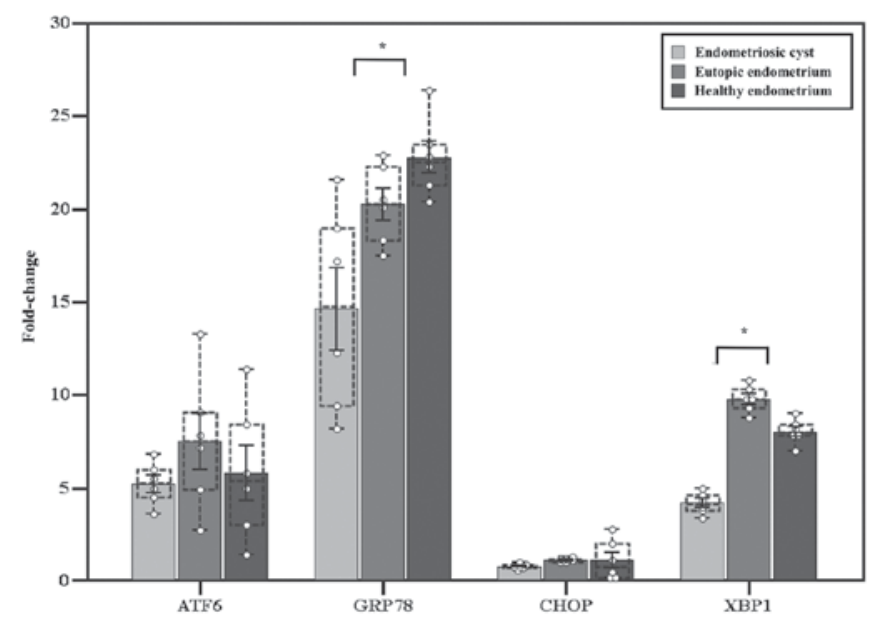

Figure 2. Gene expression analysis of UPR genes in endometriosic cysts, eutopic endometrium and healthy endometrium. Six patients with endometriosis cysts (cysts and eutopic endometrium) and six healthy patients. The solid error bars express the mean \pm standard error of the mean. The dotted error bars indicate the median, the 25 and 75 th percentiles and the lowest and highest values. Kruskal-Wallis test; ${ }^{*} \mathrm{P}<0.05$. UPR, unfolded protein response; SEM, standard error of the mean.

differences between them $(\mathrm{P}=0.51)$. GRP78 was more highly expressed in endometrial-derived tissues $(14.6 \pm 2.2, \mathrm{P}=0.03$; $20.3 \pm 0.9, \mathrm{P}=0.03$; and $22.8 \pm 0.9, \mathrm{P}=0.03$, respectively), with a significantly higher expression in endometrial tissue compared with endometriotic cysts $(\mathrm{P}=0.01)$. XBP1 s was also overexpressed $(4.2 \pm 0.2, \mathrm{P}=0.03 ; 9.8 \pm 0.3, \mathrm{P}=0.03$; and $8.0 \pm 0.3, \mathrm{P}=0.03$, respectively), with a higher level of expression in endometrial tissue compared with endometriotic cysts $(\mathrm{P}<0.001)$. CHOP expression was similar among all examined tissues $(0.8 \pm 0.1, \mathrm{P}=0.06 ; 1.4 \pm 0.1, \mathrm{P}=0.13$; and $1.1 \pm 0.4, \mathrm{P}=0.84$, respectively) (Fig. 2).

\section{Discussion}

In the present pilot study, we observed a difference in UPR gene expression between endometrioid ovarian carcinoma and healthy ovarian tissue. More specifically, ATF6 and GRP78 were more highly expressed in endometrioid ovarian carcinoma, while the expression of CHOP and XBP1 was lower. The simultaneous increase of ATF6 and GRP78 in cancer samples is in line with the current literature $(15,19-21)$. Indeed, several studies indicated that, among the different overexpressed proteins following UPR activation, GRP78 plays a crucial role in tumor proliferation, survival, metastasis and resistance to a wide variety of treatments $(19,22-24)$. The reduction of CHOP expression also reflects an important fact: Although its function in oncogenesis has not yet been clearly determined, a number of studies demonstrated that $\mathrm{CHOP}$ induction in response to prolonged ER stress causes apoptosis of pre-malignant cells, thus preventing tumor progression (25). Therefore, reduced CHOP expression, observed in endometrioid ovarian carcinoma, would be expected, as the reduction of apoptosis is a prerequisite for malignant transformation and tumor progression.

The role of XBP1 in neoplastic transformation has not yet been fully elucidated, although its oncosuppressive role appears to be predominant. While some studies highlighted the importance of the IRE1/XBP1 axis for cell survival in hypoxic environment and tumor growth $(26,27)$, others reported that several tumor types harbored mutations of IRE1 $\alpha$, leading to loss of kinase and/or endo-ribonuclease activity, splicing inhibition and reduction of XBP1, and promoting tumorigenesis (28-30). Thus, the decreased expression of XBP1 detected in endometrioid cancer is in line with a consistent part of the literature.

In summary, the alterations in the expression of UPR genes observed in endometrioid carcinoma (increased expression of ATF6 and GRP78, and reduced expression of CHOP and XBP1) appear to promote cell survival pathways and tumor progression.

Considering the association of endometrioid cancer with endometriosis, supported by years of epidemiological research $(4,5)$ and by accumulating evidence supporting that this histotype of ovarian cancer arises from endometriosis cells localized in the ovary rather than ovarian cells, it is possible to hypothesize that even endometriotic cysts may harbor UPR gene alterations that may be involved in the neoplastic progression to endometrioid ovarian cancer. In order to test this hypothesis, we compared the UPR gene expression pattern between endometriotic cysts and endometrioid carcinoma, using healthy ovarian tissue as reference.

The endometriotic cysts exhibited a simultaneous increase in ATF6 and GRP78, no difference in CHOP expression and overexpression of XBP1 in comparison with the healthy ovary; this UPR gene expression pattern was partly different from that of endometrioid cancer. More specifically, while ATF6 expression was similar between the two tissues, GRP78 was more highly expressed in endometriosis compared with endometrioid carcinoma. Conversely, CHOP expression tended to decrease in ovarian cancer compared with that in both endometriotic cysts and healthy ovarian tissue, which was in line with the role of CHOP demonstrated in different tumor models and discussed above. Finally, the expression of XBP1 was notably higher in endometriotic cysts compared with endometrioid ovarian carcinoma. The latter two differences in the expression of CHOP and XBP1 are compatible with neoplastic transformation of endometriotic cysts and may be acquired following ovarian localization of ectopic endometrial cells. However, the first two differences appear to indicate increased anti-apoptotic activity in endometriotic cysts, which 
may be partly maintained in endometrioid carcinoma, but may also be present in eutopic endometrial cells and be implicated in their migration to the ovary $(5,31)$.

In order to investigate when the alterations of UPR genes are acquired in the pathogenetic process from eutopic endometrium to endometriotic cysts and endometrioid ovarian carcinoma, and whether they result from migration outside the uterus or are physiologically expressed in the analyzed tissues, we compared their expression pattern within endometriotic cysts, eutopic endometrium and healthy endometrium using healthy ovarian tissue as reference.

We noted a greater basal UPR activation in endometrial tissue (except for CHOP) compared with ovarian tissue, which may be due to an innate diversity of the analyzed tissues. It is well known that UPR is constitutively active in cells with secretory functions and subjected to hormonal stimulation, such as endometrial cells (32-35).

No difference in CHOP expression was observed between endometriotic cysts, eutopic endometrium, healthy endometrium and healthy ovary, while it was reduced only in endometrioid ovarian cancer, confirming the oncosuppressive role of CHOP, as previously described; therefore, alterations of this gene appear to be acquired at a late stage of neoplastic progression, after the ovarian localization of ectopic endometrial cells.

XBP1 exhibited a significantly higher expression in eutopic and healthy endometrial tissue in comparison to endometriotic cysts. Conclusively, XBP1 has a high baseline expression in healthy endometrium, being a secretory tissue, it then gradually decreases in endometriosis and, to a higher degree, in ovarian carcinoma. Since the exact role of XBP1 is not clearly defined in the literature, it is important to further investigate its potential oncosuppressive role, considering that its reduction has been previously demonstrated in other tumor models.

In conclusion, our results support the hypothesis that alterations in the UPR genes CHOP and XBP1 are involved in the neoplastic progression of endometrioid ovarian cancer and are acquired following ovarian localization of ectopic endometrial cells. The evidence indicating that ATF6 and GRP78 are not increased in ovarian carcinoma compared with endometriosis does not allow considering these two genes as being directly involved in neoplastic transformation from endometriosis to endometrioid ovarian carcinoma. However, the reduction of CHOP in ovarian carcinoma, characterized by reduction of apoptosis, confirms its pro-apoptotic role. Conversely, XBP1 is overexpressed in endometrial tissues, which are secretory tissues, and is gradually reduced in endometriosis and, even more considerably, in endometrioid ovarian carcinoma, further supporting the concept of XBP1 as a marker of neoplastic transformation. Elucidating these mechanisms may represent an important step for a better understanding of cancer pathogenesis and for the development of customized therapies in the future.

The results of this pilot study are encouraging and may lay the foundation for the design of future studies with larger samples, a wider spectrum of UPR genes, and using additional methodologies such as western blotting and immunohistochemistry, in order to confirm the obtained results and achieve a better understanding of the pathogenesis of endometriosis cysts and endometrioid ovarian carcinoma.

\section{Acknowledgements}

Not applicable.

\section{Funding}

No funding was received.

\section{Availability of data and materials}

The datasets used and/or analysed during the current study are available from the corresponding author on reasonable request.

\section{Authors' contributions}

$\mathrm{AC}$ and FS conceived the study and revised the final version of the manuscript. GDC, MS and AT collected the data and drafted the manuscript. FL and EDL performed the experiments. All authors read and approved the final manuscript.

\section{Ethics approval and consent to participate}

All participants provided signed informed consent granting permission for tissue sampling and data collection. The study protocol was approved by the Marche Regional Ethics Committee of Riuniti di Ancona Hospital, Italy.

\section{Patient consent for publication}

Not applicable.

\section{Competing interests}

The authors declare that they have no competing interests.

\section{References}

1. Buggio L, Somigliana E, Barbara G, Frattaruolo MP and Vercellini P: Oral and depot progestin therapy for endometriosis: Towards a personalized medicine. Expert Opin Pharmacother 18: 1569-1581, 2017.

2. Giudice LC: Clinical practice. Endometriosis. N Engl J Med 362 : 2389-2398, 2010.

3. Kennedy S, Bergqvist A, Chapron C, D'Hooghe T, Dunselman G, Greb R, Hummelshoj L, Prentice A and Saridogan E; ESHRE Special Interest Group for Endometriosis and Endometrium Guideline Development Group: ESHRE guideline for the diagnosis and treatment of endometriosis. Hum Reprod 20: 2698-2704, 2005.

4. Giudice LC and Kao LC: Endometriosis. Lancet 364: 1789-1799, 2004.

5. Pavone ME and Lyttle BM: Endometriosis and ovarian cancer: Links, risks and challenges faced. Int J Womens Health 7: 663-672, 2015.

6. Donnez J, Nisolle M, Gillet N, Smets M, Bassil S and CasanasRoux F: Large ovarian endometriomas. Hum Reprod 11: 641-646, 1996.

7. Waller KG, Lindsay P, Curtis P and Shaw RW: The prevalence of endometriosis in women with infertile partners. Eur J Obstet Gynecol Reprod Biol 48: 135-139, 1993.

8. Sampson J: Endometrial carcinoma of the ovary arising in endometrial tissue of that organ. Arch Surg 10: 1-72, 1925.

9. Kokcu A: Relationship between endometriosis and cancer from current perspective. Arch Gynecol Obstet 284: 1473-1479, 2011.

10. Dorner AJ, Wasley LC and Kaufman RJ: Increased synthesis of secreted proteins induces expression of glucose-regulated proteins in butyrate-treated Chinese hamster ovary cells. J Biol Chem 264: 20602-20607, 1989. 
11. Poole EM, Lin WT, Kvaskoff M, De Vivo I, Terry KL and Missmer SA: Endometriosis and risk of ovarian and endometrial cancers in a large prospective cohort of U.S. nurses. Cancer Causes Control 28: 437-445, 2017.

12. Dubeau L and Drapkin R: Coming into focus: The nonovarian origins of ovarian cancer. Ann Oncol 24 (Suppl 8): viii28-viii35, 2013.

13. Prat J: New insights into ovarian cancer pathology. Annals of Oncology 23 (Suppl 10): x111-x117, 2012.

14. Schröder M and Kaufman RJ: The mammalian unfolded protein response. Annu Rev Biochem 74: 739-789, 2005.

15. Rutkowski DT, Arnold SM, Miller CN, Wu J, Li J, Gunnison KM, Mori K, Sadighi Akha AA, Raden D and Kaufman RJ: Adaptation to ER stress is mediated by differential stabilities of pro-survival and pro-apoptotic mRNAs and proteins. PLoS Biol 4: e374, 2006

16. Prat J; FIGO Committee on Gynecologic Oncology: FIGO's staging classification for cancer of the ovary, fallopian tube and peritoneum: Abridged republication. J Gynecol Oncol 26: 87-89, 2015.

17. Ledermann JA, Raja FA, Fotopoulou C, Gonzalez-Martin A, Colombo N and Sessa C; ESMO, Guidelines Working Group: Newly diagnosed and relapsed epithelial ovarian carcinoma: ESMO Clinical Practice Guidelines for diagnosis, treatment and follow-up. Ann Oncol 24 (Suppl 6): vi24-vi32, 2013.

18. Schmittgen TD and Livak KJ: Analyzing real-time PCR data by the comparative C(T) method. Nat Protoc 3: 1101-1108, 2008.

19. Bifulco G, Miele C, Di Jeso B, Beguinot F, Nappi C, Di Carlo C Capuozzo S, Terrazzano $G$, Insabato $L$ and Ulianich $L$ : Endoplasmic reticulum stress is activated in endometrial adenocarcinoma. Gynecol Oncol 125: 220-225, 2012.

20. Shuda M, Kondoh N, Imazeki N, Tanaka K, Okada T, Mori K, Hada A, Arai M, Wakatsuki T, Matsubara O, et al: Activation of the ATF6, XBP1 and grp78 genes in human hepatocellular carcinoma: A possible involvement of the ER stress pathway in hepatocarcinogenesis. J Hepatol 38: 605-614, 2003.

21. Feldman DE, Chauhan V and Koong AC: The unfolded protein response: A novel component of the hypoxic stress response in tumors. Mol Cancer Res 3: 597-605, 2005.

22. Jamora C, Dennert G and Lee AS: Inhibition of tumor progression by suppression of stress protein GRP78/BiP induction in fibrosarcoma B/C10ME. Proc Natl Acad Sci USA 93: 7690-7694, 1996.

23. Bernstein H, Payne CM, Bernstein C, Schneider J, Beard SE and Crowley CL: Activation of the promoters of genes associated with DNA damage, oxidative stress, ER stress and protein malfolding by the bile salt, deoxycholate. Toxicol Lett 108: 37-46, 1999.

24. Reddy RK, Mao C, Baumeister P, Austin RC, Kaufman RJ and Lee AS: Endoplasmic reticulum chaperone protein GRP78 protects cells from apoptosis induced by topoisomerase inhibitors: Role of ATP binding site in suppression of caspase-7 activation. J Biol Chem 278: 20915-20924, 2003.
25. Huber AL, Lebeau J, Guillaumot P, Pétrilli V, Malek M, Chilloux J, Fauvet F, Payen L, Kfoury A, Renno T, et al: p5 $8^{\mathrm{IPK}}$-mediated attenuation of the proapoptotic PERK-CHOP pathway allows malignant progression upon low glucose. Mol Cell 49: 1049-1059, 2013.

26. Romero-Ramirez L, Cao H, Nelson D, Hammond E, Lee AH, Yoshida H, Mori K, Glimcher LH, Denko NC, Giaccia AJ, et al: $\mathrm{XBP} 1$ is essential for survival under hypoxic conditions and is required for tumor growth. Cancer Res 64: 5943-5947, 2004.

27. Fujimoto T, Yoshimatsu K, Watanabe K, Yokomizo H, Otani T, Matsumoto A, Osawa G, Onda M and Ogawa K: Overexpression of human X-box binding protein 1 (XBP-1) in colorectal adenomas and adenocarcinomas. Anticancer Res 27: 127-131, 2007.

28. Ghosh R, Wang L, Wang ES, Perera BG, Igbaria A, Morita S, Prado K, Thamsen M, Caswell D, Macias H, et al: Allosteric inhibition of the IRE1 $\alpha$ RNase preserves cell viability and function during endoplasmic reticulum stress. Cell 158: 534-548, 2014.

29. Niederreiter L, Fritz TM, Adolph TE, Krismer AM, Offner FA, Tschurtschenthaler M, Flak MB, Hosomi S, Tomczak MF, Kaneider NC, et al: ER stress transcription factor Xbp1 suppresses intestinal tumorigenesis and directs intestinal stem cells. J Exp Med 210: 2041-2056, 2013.

30. Zhu H, Abulimiti M, Liu H, Su XJ, Liu CH and Pei HP: RITA enhances irradiation-induced apoptosis in p53-defective cervical cancer cells via upregulation of IRE1 $\alpha / \mathrm{XBP} 1$ signaling. Oncol Rep 34: 1279-1288, 2015.

31. Bulun SE: Endometriosis. N Engl J Med 360: 268-279, 2009.

32. Shaffer AL, Shapiro-Shelef M, Iwakoshi NN, Lee AH, Qian SB, Zhao H, Yu X, Yang L, Tan BK, Rosenwald A, et al: XBP1, downstream of Blimp-1, expands the secretory apparatus and other organelles and increases protein synthesis in plasma cell differentiation. Immunity 21: 81-93, 2004.

33. Sengupta S, Sharma CG and Jordan VC: Estrogen regulation of $\mathrm{X}$-box binding protein-1 and its role in estrogen induced growth of breast and endometrial cancer cells. Horm Mol Biol Clin Investig 2: 235-243, 2010.

34. Bruner-Tran KL, Herington JL, Duleba AJ, Taylor HS and Osteen KG: Medical management of endometriosis: Emerging evidence linking inflammation to disease pathophysiology. Minerva Ginecol 65: 199-213, 2013.

35. Burney RO, Talbi S, Hamilton AE, Vo KC, Nyegaard M, Nezhat CR, Lessey BA and Giudice LC: Gene expression analysis of endometrium reveals progesterone resistance and candidate susceptibility genes in women with endometriosis. Endocrinology 148: 3814-3826, 2007. 